ANNALS OF “DUNAREA DE JOS” UNIVERSITY OF GALATI

MATHEMATICS, PHYSICS, THEORETICAL MECHANICS

FASCICLE II, YEAR XIII (XLIV) 2021, No. 1

DOI: https://doi.org/10.35219/ann-ugal-math-phys-mec.2021.1.04

\title{
Molecular descriptors - an useful tool for assessing the physico-chemical properties of hallucinogenic drugs of abuse
}

\author{
Adelina Ion ${ }^{1, *}$, Mirela Praisler ${ }^{1,2}$, Steluta Gosav ${ }^{1,2}$ \\ I "Dunarea de Jos" University of Galati, Faculty of Sciences and Environment, 47 Domneasca Street, 800008, \\ Galati, Romania, \\ 2 "Dunarea de Jos" University of Galati, Faculty of Sciences and Environment, INPOLDE research center, \\ 800008 Galati, Romania \\ *Corresponding author: Adelina.Ion@ugal.ro
}

\begin{abstract}
Molecular descriptors play a fundamental role in chemistry and pharmaceutical sciences, being defined as a way to transform molecules into a set of numbers, allowing the mathematical assessment of the chemical information characterizing a molecule. This study presents the usefulness of molecular descriptors for the determination of physico-chemical properties of a series of new hallucinogenic amphetamines. Molecular descriptors of these compounds was performed with the Dragon 5.5 software. The chemometric processing of the information allowed the determination of structural correlations (similarities) between this class of designer drugs and an unknown compound.
\end{abstract}

Keywords: Hallucinogenic amphetamines, molecular descriptors.

\section{INTRODUCTION}

According to R. Todeschini and V. Consonni, molecular descriptors are mathematical representations of a molecule obtained by a well-specified algorithm, applied to a well-defined molecular representation or a well-specified experimental procedure [1]. These descriptors are numerical values that characterize different properties of molecules and can be used to determine a large variety of quantitative structure-activity relationship (QSAR) or the quantitative structureproperty relationship (QSPR). As molecular descriptors are important elements that provide information about the molecular structure, they are useful for describing and classifying the structure of a chemical compound, its structure being associated with its properties. On this basis, the chemical and biological properties of a compound can be predicted if the structure is well known and represented. Many scientific papers prove that molecular descriptors contain relevant information about the type of atoms and functional groups present in the molecular structure of the analyzed compound, as well as about the molecular size and shape, indices of molecular connectivity, spatial distribution of molecules, etc. [2-5].

This study presents the physico-chemical characterization of the molecular structure of a series of recently discovered hallucinogenic amphetamines, i.e. 3,4-methylenedioxymethamphetamine (C1); 7-(2-aminopropyl)benzofuran (C2); 5,6-methylenedioxy-2-aminoindane $\quad$ (C3); 3,4-Methylenedioxypyrovalerone (C4); 1-(benzofuran-5-yl)- $N$-methylpropan-2-amine) (C5); N-Ethyl4-methoxyamphetamine (C6); N-ethylpentylone (C7). 


\section{EXPERIMENTAL}

The molecular structures of all the above mentioned compounds were represented in 3D coordinates, and their geometries were optimized with the semi-empirical method AM1 (by using the HyperChem 8.03 software [6]), as well as with the DFT quantum method (by using the Gaussian 09 software [7]).

Four QSAR descriptors were determined for each studied compound. Three of these were obtained directly from the Gaussian program .log output file, namely: the energy of the lowest unoccupied molecular orbital $\left(E_{L U M O}\right)$, the energy of the highest occupied molecular orbital $\left(E_{\text {НОмO }}\right)$, the dipole moment $(D M)$. The fourth descriptor, the $E_{\text {gap }}$ energy, was calculated using the following formula:

$$
E_{\text {gap }}=E_{\text {LUMO }}-E_{\text {НОМO }}
$$

The dipole moment $D M$ is an electronic parameter, i.e. a measure of the polarization of the system (the separation of positive and negative electric charges from a system of electric charges). The HOMO molecular orbital acts mainly as an electron donor, while the LUMO molecular orbital acts mainly as an electron acceptor. $E_{g a p}$ characterizes the chemical stability/reactivity of the compound as a measure of the conductivity of electrons.

The study continued by determining several types of molecular descriptors, by using the Dragon 5.5 program [8]. The .hin files obtained by using the Hyperchem 8.03 program, which contains the optimized structures, were used as input files for the Dragon 5.5 program. The latter calculated a series of descriptors such as charge descriptors, constitutional descriptors, and some molecular properties.

\section{RESULTS AND DISCUSSION}

The results obtained for the four QSAR molecular descriptors calculated for the seven hallucinogenic amphetamines of interest are presented in Table 1.

Table 1. Molecular QSAR descriptors calculated for C1, C2, C2, C4, C5, C6, and C7 hallucinogenic amphetamines [9].

\begin{tabular}{|c|c|c|c|c|}
\hline \multirow{2}{*}{$\begin{array}{c}\text { Chemical } \\
\text { compound }\end{array}$} & \multicolumn{4}{|c|}{ QSAR descriptors } \\
\cline { 2 - 5 } & $\begin{array}{c}D M \\
(\text { Debye })\end{array}$ & $\begin{array}{c}E_{L U M O} \\
(\mathrm{eV})\end{array}$ & $\begin{array}{c}E_{H O M O} \\
(\mathrm{eV})\end{array}$ & $\begin{array}{c}E_{g a p} \\
(\mathrm{eV})\end{array}$ \\
\hline C1 & 1.54 & -1.54 & -5.37 & 3.83 \\
\hline C2 & 1.95 & -0.60 & -6.01 & 5.41 \\
\hline C3 & 1.36 & -0.31 & -5.41 & 5.10 \\
\hline C4 & 3.72 & -0.78 & -5.61 & 4.83 \\
\hline C5 & 1.65 & -0.80 & -5.88 & 5.08 \\
\hline C6 & 1.65 & -0.37 & -5.83 & 5.46 \\
\hline C7 & 2.94 & -1.48 & -5.97 & 4.53 \\
\hline
\end{tabular}


Charge descriptors are electronic descriptors defined in terms of atomic charges and used to describe the electronic aspects of the entire molecule and particular regions, such as atoms, bonds, and molecular fragments. Constitutional descriptors are descriptors categorized as simple (0D) and they reflect the molecular composition of a compound without requiring any information about its molecular geometry. The values of these descriptors and the associated molecular parameters determined for the analyzed drugs of abuse are presented in Table 2 [9].

Table 2. The charge and constitutional descriptors, as well as the molecular parameters calculated for each of the seven hallucinogenic amphetamines included in the database.

\begin{tabular}{|c|c|c|c|c|c|c|c|c|}
\hline \multirow{2}{*}{$\begin{array}{l}\text { Descriptor } \\
\text { class }\end{array}$} & \multirow{2}{*}{$\begin{array}{l}\text { Descriptor } \\
\text { code }\end{array}$} & \multicolumn{7}{|c|}{ Chemical compounds } \\
\hline & & C1 & $\mathrm{C2}$ & $\mathbf{C 3}$ & $\mathrm{C} 4$ & C5 & C6 & C7 \\
\hline \multirow{10}{*}{ Charge } & qpmax & 0,233 & 0,135 & 0,233 & 0,233 & 0,135 & 0,122 & 0,233 \\
\hline & qnmax & $-0,453$ & $-0,463$ & $-0,453$ & $-0,453$ & $-0,463$ & $-0,495$ & $-0,453$ \\
\hline & Qpos & 1,42 & 1,035 & 1,359 & 1,785 & 1,039 & 1,125 & 1,78 \\
\hline & Qneg & $-1,42$ & $-1,035$ & $-1,359$ & $-1,785$ & $-1,039$ & $-1,125$ & $-1,78$ \\
\hline & Qtot & 2,841 & 2,07 & 2,717 & 3,57 & 2,078 & 2,25 & 3,56 \\
\hline & Qmean & 0,109 & 0,08 & 0,113 & 0,087 & 0,072 & 0,068 & 0,096 \\
\hline & $\mathbf{Q 2}$ & 0,708 & 0,42 & 0,7 & 0,799 & 0,405 & 0,434 & 0,817 \\
\hline & RPCG & 0,164 & 0,13 & 0,172 & 0,131 & 0,13 & 0,109 & 0,131 \\
\hline & RNCG & 0,319 & 0,447 & 0,333 & 0,254 & 0,446 & 0,44 & 0,254 \\
\hline & SPP & 0,686 & 0,598 & 0,686 & 0,686 & 0,598 & 0,617 & 0,686 \\
\hline \multirow{10}{*}{ Constitutional } & MW & 179,24 & 175,25 & 177,22 & 275,38 & 189,28 & 193,32 & 249,34 \\
\hline & AMW & 6,89 & 6,74 & 7,38 & 6,72 & 6,53 & 5,86 & 6,74 \\
\hline & Sv & 15,6 & 16,09 & 15,01 & 25,1 & 17,69 & 18,89 & 21,91 \\
\hline & Se & 26,06 & 25,73 & 24,17 & 40,92 & 28,61 & 32,38 & 37,04 \\
\hline & Sp & 16,48 & 17,03 & 15,72 & 25,98 & 18,79 & 20,31 & 23,22 \\
\hline & Mv & 0,6 & 0,62 & 0,63 & 0,6 & 0,61 & 0,57 & 0,59 \\
\hline & Me & 1 & 0,99 & 1,01 & 1 & 0,99 & 0,98 & 1 \\
\hline & Мp & 0,63 & 0,65 & 0,66 & 0,63 & 0,65 & 0,62 & 0,63 \\
\hline & ARR & 0,429 & 0,714 & 0,4 & 0,273 & 0,667 & 0,429 & 0,316 \\
\hline & nBnz & 1 & 1 & 1 & 1 & 1 & 1 & 1 \\
\hline \multirow{10}{*}{$\begin{array}{l}\text { Molecular } \\
\text { properties }\end{array}$} & Hy & 0,53 & 0,455 & 0,53 & $-0,787$ & $-0,305$ & $-0,305$ & $-0,28$ \\
\hline & AMR & 49,471 & 52,545 & 48,032 & 76,891 & 57,32 & 59,69 & 68,804 \\
\hline & TPSA(Tot) & 44,48 & 39,16 & 44,48 & 38,77 & 25,17 & 21,26 & 47,56 \\
\hline & ALOGP & 1,403 & 1,938 & 1,129 & 3,376 & 2,37 & 2,399 & 2,728 \\
\hline & MLOGP & 1,379 & 1,866 & 1,379 & 2,362 & 2,147 & 2,505 & 1,856 \\
\hline & Hypnotic-80 & 1 & 1 & 1 & 1 & 1 & 1 & 1 \\
\hline & Depressant80 & 0 & 0 & 0 & 1 & 0 & 0 & 1 \\
\hline & BLTF96 & $-2,56$ & $-2,98$ & $-2,56$ & $-3,4$ & $-3,21$ & $-3,52$ & -2.97 \\
\hline & BLTD48 & $-2,63$ & $-3,09$ & $-2,63$ & $-3,56$ & $-3,36$ & $-3,7$ & $-3,08$ \\
\hline & BLTA96 & $-2,61$ & $-3,1$ & $-2,61$ & $-3,59$ & $-3,38$ & $-3,73$ & $-3,09$ \\
\hline
\end{tabular}




\section{CONCLUSIONS}

The results indicate that the most representative molecular descriptors are the molecular weight (MW), Moriguchi octanol-water partition coefficient (MLOGP), Ghose-Crippen octanol-water partition coefficient (ALOGP), hydrophilic factor (Hy), maximum positive and negative charge (qpmax and qnmax, respectively), polar topological surface area (TPSA), Verhaar model of Fish baseline toxicity from MLOGP (mmol/l) (BLTF96), Verhaar model of Daphnia base-line toxicity from MLOGP (mmol/l) (BLTD48) and the Verhaar model of Algae base-line toxicity from MLOGP (mmol/l) (BLTA96). The $\log P$ represents the value of the octanol/water partition coefficient, representing at the same time a measure of the hydrophilicity of the chemical compound. A low LogP means an absorption or a high membrane permeability of the chemical compound in the human body, i.e. a high hydrophilicity.

The results indicate that the highest hydrophilicity is found for the hallucinogenic amphetamines $\mathrm{C} 1(0.53)$ and $\mathrm{C} 3(0.53)$, which are characterized by the lowest values for ALOGP (1.403 for compound $\mathrm{C} 1$ and 1.129 for $\mathrm{C} 3$, respectively), and for MLOGP (1,379 for compounds C1 and C3). Therefore, the hallucinogenic amphetamines $\mathrm{C} 1$ (3,4-methylenedioxymethamphetamine) and C3 (5,6-methylenedioxy-2-aminoindane) have the highest cellular permeability among the compounds analyzed.

Regarding toxicological activity, the results show that it is necessary to evaluate the toxicities of BLTF96 and BLTA96 for the studied hallucinogenic amphetamines, which exert a strong toxic effect on humans and are also dangerous for the environment. The toxicity ranges between 2.56 and 3.52 for BLTF96, and between -2.61 and -3.59 for BLTA96.

\section{References}

1. Todeschini R., Consonni V., Handbook of Molecular Descriptors, Wiley-VCH, 2000.

2. Danishuddin Khan A.U., Descriptors and their selection methods in QSAR analysis: paradigm for drug design, Drug Discovery Today 21 (2016) 1291-1302.

3. Toropova A.P., Schultz T.W., Toropov A.A., Building up a QSAR model for toxicity toward Tetrahymena pyriformis by the Monte Carlo method: A case of benzene derivative, Environmental Toxicology and Pharmacology 42 (2016) 135-145.

4. Roy K., Kar S., Das R.N., Understanding the Basics of QSAR for Applications in Pharmaceutical Sciences and Risk Assessment. Background of QSAR and Historical Developments. In: KRKN Das, editor. Boston: Academic Press; 1-46, 2015.

5. Kar S., Roy K., QSAR of phytochemicals for the design of better drugs. Expert Opinion on Drug Discovery 7 (2012) 877-902.

6. Hyperchem software, Version 8.0.3., Hyper Co., USA, 2007.

7. Gaussian 09 software package, Revision D.01, Frisch M. J. et al., Gaussian, Inc., Wallingford CT, 2009.

8. Talete S. R. L., DRAGON for Windows (Software for Molecular Descriptors Calculation), Version 5.5, Milano - Italy, 2007.

9. https://www.ugal.ro/files/doctorat/sustineri/2020/AdelinaION_PHD_030820.pdf 